\title{
A near-field scanning optical microscopy study of the uniformity of GaAs surface passivation
}

\author{
Jutong Liu and T. F. Kuech \\ Department of Chemical Engineering, University of Wisconsin-Madison, Madison, Wisconsin 53706
}

(Received 22 February 1996; accepted for publication 21 May 1996)

\begin{abstract}
We have achieved spatially resolved photoluminescence (PL) from metalorganic vapor phase epitaxy (MOVPE) grown GaAs surfaces by near-field scanning optical microscopy (NSOM). We have performed the topography, reflection, and PL measurements by NSOM combined with the topography measurements by atomic force microscopy (AFM) on the as-grown and $\left(\mathrm{NH}_{4}\right)_{2} \mathrm{~S}$-passivated $\mathrm{GaAs}$ samples. The uniformity of $\mathrm{GaAs}$ with a thin $\mathrm{Al}_{0.65} \mathrm{Ga}_{0.35} \mathrm{As}$ cap layer has also been studied and compared with the $\left(\mathrm{NH}_{4}\right)_{2} \mathrm{~S}$ treated samples. We found the submicron scale variations in PL intensity which were not correlated to the topographic features. The PL intensity variation was related to the changes in the surface state density. Semiquantitative analyses of the resolution limits of NSOM-based PL measurements and surface state variations are presented. (C) 1996 American Institute of Physics. [S0003-6951(96)03731-X]
\end{abstract}

Most as-grown III-V semiconductor surfaces possess a very high surface state density $\left(N_{s} \sim 10^{12} \mathrm{~cm}^{-2}\right)$, particularly after exposure to air. ${ }^{1}$ These surface states are nonradiative recombination centers which impact the performance of many minority carrier devices, such as light emitting diodes, solar cells, heterojunction bipolar transistors, and lasers. This high density of surface states leads to Fermi level pinning near-midgap, resulting in difficulties in the Ohmic contact formation and in the fabrication of metal-insulatorsemiconductor field-effect transistors (MISFET). ${ }^{2}$ In recent years, many studies have focused on the effective passivation of the compound semiconductor surfaces. ${ }^{3}\left(\mathrm{NH}_{4}\right)_{2} \mathrm{~S}$ passivation of GaAs surfaces has been proven to be very successful and easy to implement. ${ }^{4}$

Several characterization techniques have been used to probe the properties of the passivated surfaces. ${ }^{5,6}$ The uniformity of passivation at submicron scale, however, has rarely been studied. As the device dimensions decrease to the nanoscale, the surface properties and uniformity of these properties at the submicron scale become increasingly important. ${ }^{7}$ Near-field scanning optical microscopy (NSOM) has been demonstrated to be a powerful tool for performing optical measurements at a much higher spatial resolution than conventional far-field optics. ${ }^{8}$ In this work, the spatially resolved PL measurements on the passivated GaAs surfaces, using NSOM, are presented. These quantitative measurements provide information on the surface state density variation as well as the independently determined surface morphologies of the passivated surface. Measurements of the optical uniformity of $\left(\mathrm{NH}_{4}\right)_{2} \mathrm{~S}$-passivated surfaces are compared to control samples consisting of a thin $\mathrm{Al}_{0.65} \mathrm{Ga}_{0.35} \mathrm{As}$ cap layer on an epitaxial GaAs layer. This structure serves as an alternative GaAs surface passivation. The epitaxially grown $\mathrm{GaAs}-\mathrm{Al}_{x} \mathrm{Ga}_{1-x} \mathrm{As}$ heterostructure has a low interface state density and interface recombination velocity ${ }^{9,10}$ and should have more uniform properties than the free GaAs surface.

The NSOM is a home-built system with a commercial scanning control unit. ${ }^{11}$ All the experiments are done in illumination mode where the NSOM tip serves as the excitation source. The optical collector is a parabolic reflector with the tip at its focal point, maximizing the collection efficiency. A low level $\mathrm{He}-\mathrm{Ne}$ laser light is used for the standard shear force distance regulation. ${ }^{12}$ Topographic and optical images are collected simultaneously with the detection wavelength fixed at the band-edge energy of GaAs $(870 \mathrm{~nm})$.

All the samples used in this study were grown by metalorganic vapor phase epitaxy (MOVPE) on GaAs (001) wafers misoriented by $2^{\circ}$ towards the nearest $\langle 110\rangle$ azimuth. The GaAs epitaxial layer was $1 \mu \mathrm{m}$ thick with $p$-type $\mathrm{Zn}$ doping of $9 \times 10^{19} \mathrm{~cm}^{-3}$. The passivation process consisted of the removal of native oxides by soaking the sample in hot $\mathrm{H}_{2} \mathrm{SO}_{4}: \mathrm{H}_{2} \mathrm{O}(1: 1)$ solution, followed by a shallow $30 \mathrm{~nm}$ etching of $\mathrm{GaAs}$ in a dilute solution of $\mathrm{H}_{2} \mathrm{SO}_{4}: \mathrm{H}_{2} \mathrm{O}_{2}: \mathrm{H}_{2} \mathrm{O}$ (1:8:500), a DI water rinse for $5 \mathrm{~min}$, treatment in a $22 \%$ solution of $\left(\mathrm{NH}_{4}\right)_{2} \mathrm{~S}$ in $\mathrm{H}_{2} \mathrm{O}$ for $5 \mathrm{~min}$ and a final DI water rinse for 5 min. $^{2}$ A control sample consisted of similarly doped epitaxial $p$-GaAs with a $3 \mathrm{~nm}$ thick $p$-type $\mathrm{Al}_{0.65} \mathrm{Ga}_{0.35} \mathrm{As}$ cap layer. This sample was used in the "asgrown' condition.

We obtained surface scans of the topographic, reflection and PL signals on the as-grown, passivated and GaAs- $\mathrm{Al}_{0.65} \mathrm{Ga}_{0.35} \mathrm{As}$ heterostructure samples. Figure 1 shows the simultaneously collected topography and reflection images from a GaAs sample after $\left(\mathrm{NH}_{4}\right)_{2} \mathrm{~S}$ surface passivation. The NSOM topography features at submicron scale are very similar to those observed by atomic force microscopy (AFM). Similar values of the rms roughness were obtained by NSOM and AFM on all samples, indicating both a stable NSOM distance regulation and a high sensitivity to subnanometer height variation. The PL image, shown in Fig. 2 , indicates luminescence variations on the submicron spatial scale that are clearly observed. The surface optical and topographic structures were analyzed through the determination of the lateral correlation functions and are presented in the figure captions. The lateral correlation lengths were determined which represent the smallest feature sizes within resolution limits of the instrument for the topographic and optical signals. ${ }^{13}$ These values indicate we have achieved submicron spatial resolution for the NSOM-based optical and topo- 

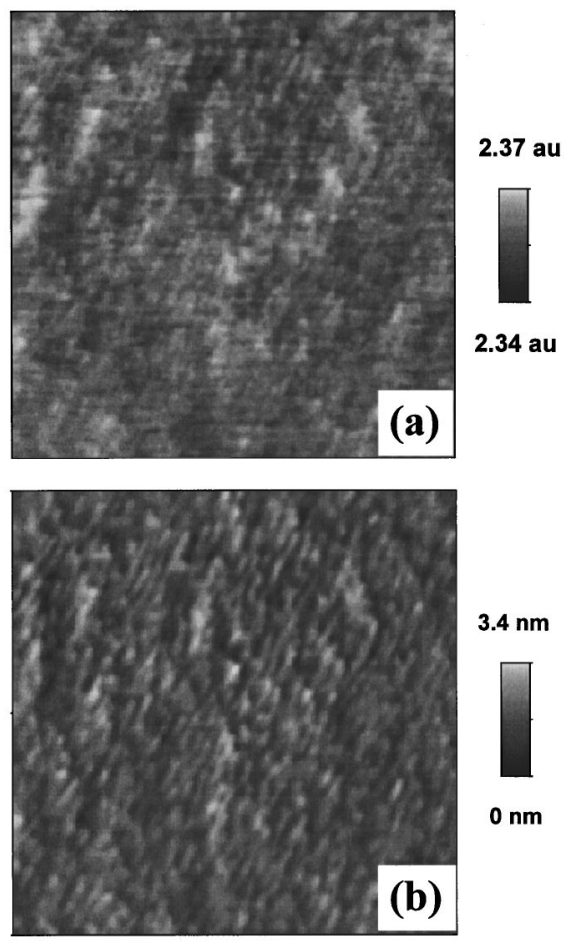

FIG. 1. Simultaneously collected $2.6 \mu \mathrm{m} \times 2.6 \mu \mathrm{m}$ (a) reflection and (b) topography signals from a GaAs sample after $\left(\mathrm{NH}_{4}\right)_{2} \mathrm{~S}$ surface passivation. A strong coupling between the reflection and topography signals is observed. The lateral correlation lengths for the two images are 75 and $39 \mathrm{~nm}$, respectively. The cross correlation coefficient for the two images is 0.59 .
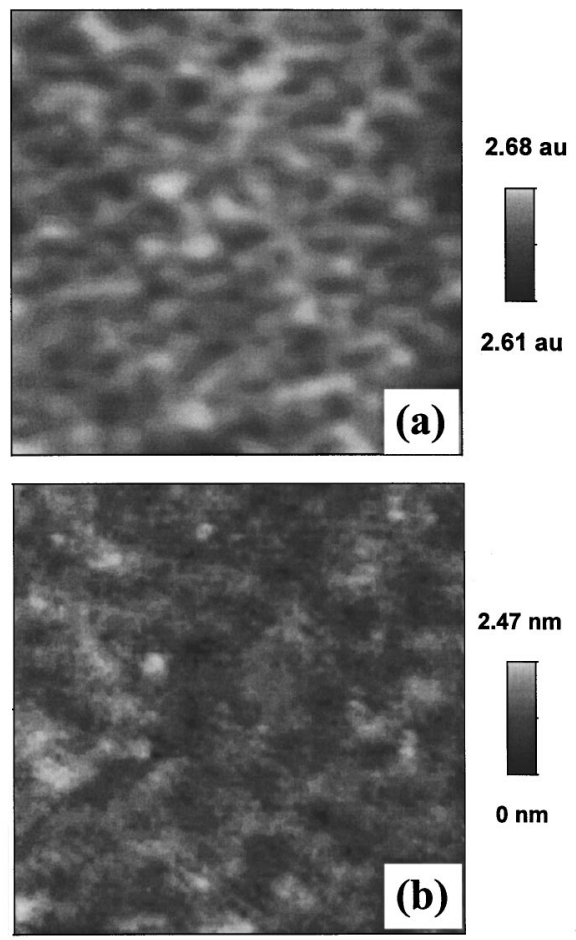

FIG. 2. Simultaneously collected $2.4 \mu \mathrm{m} \times 2.4 \mu \mathrm{m}$ PL (a) and topographic (b) signals from an as-grown GaAs surface. Negligible coupling exists between the topography and PL signals. A tip with a larger aperture was used in this scan. The lateral correlation lengths for the two images are 116 and $120 \mathrm{~nm}$, respectively. The cross correlation coefficient for the two images is -0.04 .
TABLE I. Analysis of spatially resolved PL measurements using NSOM.

\begin{tabular}{lcccc}
\hline \hline & $\Delta \mathrm{PL} \pm \sigma_{\Delta \mathrm{PL}}$ & $\Delta N_{s} \pm \sigma_{\Delta N_{s}}$ & $\begin{array}{c}\text { NSOM } \\
\text { topography } \\
\text { rms } \pm \sigma \\
(\mathrm{nm})\end{array}$ & $\begin{array}{c}\mathrm{AFM} \\
\mathrm{rms} \pm \sigma \\
(\mathrm{nm})\end{array}$ \\
\hline \multicolumn{1}{|c|}{ As-grown } & $0.86 \pm 0.05$ & $0.42 \pm 0.02$ & $0.25 \pm 0.03$ & $0.23 \pm 0.01$ \\
$\left(\mathrm{NH}_{4}\right)_{2} \mathrm{~S}$ & $0.73 \pm 0.02$ & $0.35 \pm 0.01$ & $0.24 \pm 0.01$ & $0.27 \pm 0.06$ \\
passivated & & & & \\
$\begin{array}{l}\mathrm{Al}_{0.65} \mathrm{Ga}_{0.35} \mathrm{As} \\
\text { capped }\end{array}$ & $0.59 \pm 0.06$ & $0.28 \pm 0.03$ & $0.38 \pm 0.08$ & $0.31 \pm 0.02$ \\
\hline \hline
\end{tabular}

graphic measurements. The quantitative cross-correlation coefficient analysis, ${ }^{14}$ carried out on a pixel-by-pixel basis, indicates a negligible correlation between the topography and PL signals in contrast to the strong correlation between the measured topography and reflection signals. ${ }^{15}$

A theoretical model was developed in order to relate the PL intensity variation to the change of surface state density and to estimate the spatial resolution limit. The specific surface state density and bulk doping distribution determine the band bending and the electric field within the depletion region. Assuming a typical minority carrier lifetime in the bulk of $0.1 \mathrm{~ns}$, we used the one-dimensional analytic solution of Mettler ${ }^{16}$ to calculate the functional dependence of the PL intensity on surface state density. Within this model, Mettler characterized minority carrier transport to the surface through the use of a virtual recombination velocity, $S_{v}$, at the depletion region boundary:

$$
\begin{aligned}
\mathrm{PL} & \propto(1-R) N e^{-\left(\alpha_{e}+\alpha_{p}\right) w} \frac{\alpha_{e} L_{n}}{\left(\alpha_{e} L_{n}\right)^{2}-1} \\
& \times\left(\frac{S_{v} L_{n} / D_{n}+\alpha_{e} L_{n}}{\left(S_{v} L_{n} / D_{n}+1\right)\left(\alpha_{p} L_{n}+1\right)}-\frac{1}{\left(\alpha_{e}+\alpha_{p}\right) L_{n}}\right),
\end{aligned}
$$

where $R$ is the surface reflectivity, $N$ is the excitation intensity, $\alpha_{e}$ and $\alpha_{p}$ are the absorption coefficients at the excitation and emission wavelengths, $L_{n}$ and $D_{n}$ are the diffusion length and diffusion coefficient of the minority carriers. For the highly doped samples in this study, the depletion region is so narrow, smaller than $3 \mathrm{~nm}$, that the PL from the depletion region is estimated to be less than $10^{-6}$ of the total PL. Therefore, the virtual recombination velocity, $S_{v}$, in this limit, becomes the actual surface recombination velocity $S$. Under low illumination conditions, the recombination velocity has the form, $S=N_{s} \sigma v_{t h}$, where $N_{s}$ is the surface state density, $\sigma$ is the capture cross section and $v_{t h}$ is the thermal velocity of carriers. We measure the variation in the $N_{s} \sigma$ product, since $v_{t h}$ is a constant. Because the nature of the surface states is assumed not to change with passivation, we will assume the variation in PL is due directly to the local variation in $N_{s}$, i.e., $\sigma$ is a constant.

This model was used to analyze the data derived from the NSOM with results shown in Table I, where $\Delta \mathrm{PL}$ is defined as the percentage rms variation of the PL signals. The percentage variation of surface (interface) state density $\Delta N_{s}$ is calculated from $\Delta \mathrm{PL}$ using Eq. (1). The associated error in these values is also presented in this table. The analysis shows that the surface (interface) state density variation is on the order of several percent over a $2.38 \mu \mathrm{m}$ region 

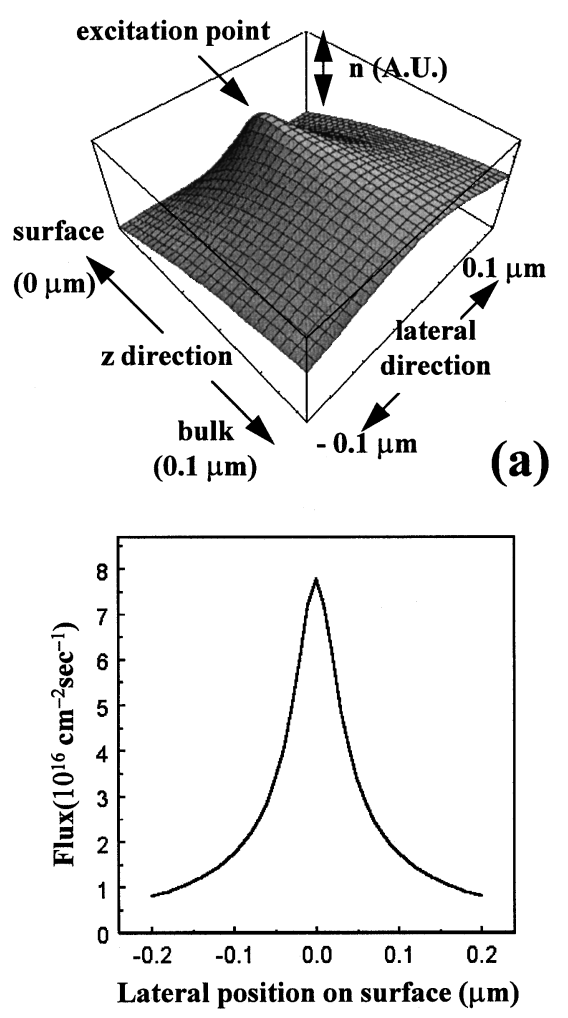

(b)

FIG. 3. The results of the numerical modeling of the localized PL obtained from $p$-type GaAs using NSOM. (a) The calculated photogenerated carrier distribution in a cross section perpendicular to the surface has a lateral decay length $(\sim 55 \mathrm{~nm})$ which is much smaller than the intrinsic diffusion length as a result of the carrier recombination at the surface. (b) The lateral plot of the carrier flux to the surface indicates a rapid drop off with an $e^{-1}$ width of $\sim 120 \mathrm{~nm}$.

for all the samples. The comparison between the state density variation for the three materials is: $\mathrm{Al}_{0.65} \mathrm{Ga}_{0.35}$ As-capped $<\left(\mathrm{NH}_{4}\right)_{2} \mathrm{~S}$-passivated $<$ As-grown. In addition to possessing the observed strongest PL intensity level, the $\mathrm{Al}_{0.65} \mathrm{Ga}_{0.35}$ As-capped sample exhibited the lowest variation in interface state density. It is expected that since the interface was produced through the epitaxial growth process, it should be more uniform than the free surface or a surface passivated by wet chemical treatment. ${ }^{9,10}$

As in all scanning probe measurements, the spatial resolution of the measured signal is of great interest. The spatial resolution limit of the localized PL experiments using NSOM can be estimated from an analytic calculation of the carrier transport and recombination in lateral directions. We ignore the depletion region and assume a point excitation source near the surface. The two-dimensional carrier transport equation was solved assuming that the surface has an infinite surface recombination velocity and recombination in the bulk is characterized by a single recombination lifetime. As shown in Fig. 3, the presence of surface states greatly narrows the lateral distribution of the photogenerated carriers, leading to the lateral resolution limit of about $120 \mathrm{~nm}$. Since the aperture size of the NSOM probe we used is less than $90 \mathrm{~nm},{ }^{11}$ the spatial resolution should be minority carrier diffusion limited. The lateral correlation length obtained from the PL image (Fig. 2) is $116 \mathrm{~nm}$, indicating it is associated with the spatial resolution of the localized PL measurements using NSOM. This calculation also indicates that the recombination rate at the surface is not saturated, even at the high excitation density used in these NSOM experiments, provided the surface recombination time constant is less than $100 \mathrm{~ns}$. Given the negligible depletion width in these measurements, the observed variation in the optical signal should therefore be directly related to the surface state density.

This work was supported by the National Science Foundation through the Materials Research Group on Chemical Vapor Deposition (MRG DMR9121074) and the Army Research Office (DAAH04-95-1-0322). The authors would like to acknowledge the assistance of J. W. Huang on the MOVPE sample growth and the helpful discussions with Dr. C. Teichert.

${ }^{1}$ H. Hasegawa and H. Ohno, J. Vac. Sci. Technol. B 4, 1130 (1986).

${ }^{2}$ M. S. Carpenter, M. R. Melloch, and T. E. Dungan, Appl. Phys. Lett. 53, 66 (1988).

${ }^{3}$ C. J. Spindt, R. S. Besser, R. Cao, K. Miyano, C. R. Helms, and W. E. Spicer, Appl. Phys. Lett. 54, 1148 (1989).

${ }^{4}$ C. J. Sandroff, R. N. Nottenburg, J.-C. Bischoff, and R. Bhat, Appl. Phys. Lett. 51, 33 (1987).

${ }^{5}$ S. Habibi, M. Totsuka, J. Tanaka, and S. Matsumoto, J. Vac. Sci. Technol. B 13, 1466 (1995).

${ }^{6}$ D. Paget, V. L. Berkovits, and A. O. Gusev, J. Vac. Sci. Technol. A 13, 2368 (1995).

${ }^{7}$ J. A. Dagata, W. Tseng, J. Bennet, E. A. Dobisz, J. Schneir, and H. H. Harary, J. Vac. Sci. Technol. A 10, 2105 (1992).

${ }^{8}$ R. D. Grober, T. D. Harris, J. K. Trautman, E. Betzig, W. Wegscheider, L. Pfeiffer, and K. West, Appl. Phys. Lett. 64, 1421 (1994).

${ }^{9}$ D. J. Wolford, G. D. Gilliland, T. F. Kuech, J. Klem, H. P. Hjalmarson, J. A. Bradley, C. F. Tsang, and J. Martinsen, Appl. Phys. Lett. 64, 1413 (1994).

${ }^{10}$ D. J. Wolford, G. D. Gilliland, T. F. Kuech, L. M. Smith, J. Martinsen, J. A. Bradley, C. F. Tsang, R. Venkatasubramanian, S. K. Ghandhi, and H. P. Hjalmarson, J. Vac. Sci. Technol. B 9, 2369 (1991).

${ }^{11}$ TopoMetrix Corporation, 5403 Betsy Ross Dr., Santa Clara, CA 950541192.

${ }^{12}$ E. Betzig, P. L. Finn, and J. S. Weiner, Appl. Phys. Lett. 60, 2484 (1992).

${ }^{13}$ H.-N. Yang, G.-C. Wang, and T.-M. Lu, Diffraction from Rough Surfaces and Dynamic Growth Fronts (World Scientific, Singapore, 1993), Chap. II.

${ }^{14}$ R. C. Gonzalez and R. E. Woods, Digital Image Processing (AddisonWesley, New York, 1992), Chap. 9.

${ }^{15}$ S. I. Bozhevolnyi, I. I. Smolyaninov, and O. Keller, Appl. Phys. Lett. 34, 3793 (1995)

${ }^{16}$ K. Mettler, Appl. Phys. Lett. 12, 75 (1977). 